\title{
Reliability of positron emission tomography-computed tomography in identification of mediastinal lymph node status in patients with non-small cell lung cancer
}

\author{
Maruf Şanl, MD, ${ }^{\mathrm{a}}$ Ahmet Feridun Isik, MD, ${ }^{\mathrm{a}}$ Sabri Zincirkeser, MD, ${ }^{\mathrm{b}}$ Osman Elbek, MD, \\ Ahmet Mete, MD, ${ }^{\mathrm{d}}$ Bulent Tuncozgur, MD, ${ }^{\mathrm{a}}$ and Levent Elbeyli, $\mathrm{MD}^{\mathrm{a}}$
}

\begin{abstract}
Objective: The involvement of mediastinal lymph nodes is a very important prognostic factor in patients with potentially resectable non-small cell lung cancer. Our aim in this study was to investigate the value of positron emission tomographic-computed tomographic scanning in staging lung cancer, especially for mediastinal lymph node evaluation, and to determine whether this could decrease the need for mediastinoscopy.
\end{abstract}

\begin{abstract}
Methods: Seventy-eight patients with non-small cell lung cancer who were potential candidates for surgical resection and admitted to the thoracic surgery unit of our hospital from March 2006 to June 2008 joined this prospective study. Positron emission tomographic-computed tomographic scanning was performed as part of the prospective studies used to diagnose or stage the tumors. All 78 patients underwent tissue sampling of mediastinal lymph nodes to compare these with imaging results. The diagnostic efficacy of the computed tomographic and positron emission tomographic-computed tomographic scans compared with histopathologic findings were calculated with sensitivity, specificity, positive and negative predictive values, and accuracy.
\end{abstract}

Results: Final histology was available on 397 lymph node stations (N1, N2, and N3) sampled from 78 patients during mediastinoscopy or surgical intervention. Sensitivity, specificity, and positive and negative predictive values of mediastinal lymph node involvement in patients undergoing thoracic computed tomographic scanning were $45.4 \%, 80.5 \%, 27.7 \%$, and $90 \%$, respectively. The accuracy of computed tomographic scanning was $75.6 \%$. The sensitivity, specificity, and positive and negative predictive values of mediastinal lymph node involvement in patients undergoing positron emission tomographic-computed tomographic scanning were $81.8 \%, 89.5 \%, 56.2 \%$, and $96.7 \%$, respectively.

Conclusion: There is a need for mediastinoscopy in positron emission tomographic-computed tomographic scanning-positive mediastinal lymph nodes, but it might not be necessary for positron emission tomographiccomputed tomographic scanning-negative lymph nodes.

The involvement of mediastinal lymph nodes (MLNs) is an important prognostic factor in patients with potentially resectable non-small cell lung cancer (NSCLC). The appropriate treatment of patients with NSCLC is based on accurate staging. Various diagnostic tools are used for preoperative staging of NSCLC, including chest radiography, computed tomography (CT), magnetic resonance imaging, bronchoscopy, thoracoscopy, mediastinoscopy, endobronchial and endoscopic ultrasound-guided fine-needle aspiration biopsy, and positron emission tomography (PET). PET scanning with [18F]-2-fluoro-2-deoxy-D-glucose (FDG) imaging has shown substantial promise during the past decade in helping with the noninvasive preoperative staging of lung cancer. ${ }^{1}$ If

From the Thoracic Surgery Department, ${ }^{\mathrm{a}}$ Nuclear Medicine Department, ${ }^{\mathrm{b}}$ Chest Disease Department, ${ }^{\mathrm{c}}$ and Radiology Department, ${ }^{\mathrm{d}}$ Gaziantep University, School of Medicine, Gaziantep, Turkey.

Received for publication Sept 1, 2008; revisions received Feb 24, 2009; accepted for publication March 23, 2009; available ahead of print June 19, 2009.

Address for reprints: Maruf Şanlı, MD, Gaziantep Üniversitesi, Tıp Fakültesi, Göğüs Cerrahisi AD, 27310-Şehitkamil/Gaziantep/Turkey (E-mail: sanli@gantep.edu.tr). J Thorac Cardiovasc Surg 2009;138:1200-5

$0022-5223 / \$ 36.00$

Copyright (c) 2009 by The American Association for Thoracic Surgery doi:10.1016/j.jtcvs.2009.03.035 there are no distant metastases, the status of the MLNs is critical. Resection is not considered in patients with lung cancer if there is MLN involvement. In these patients neoadjuvant treatment is recommended before surgical intervention. ${ }^{2}$

Although PET scanning might accurately accomplish lymph node staging in patients with lung cancer, mediastinoscopy is often used for deciding unresectability pertaining to the presence of $\mathrm{N} 2 / \mathrm{N} 3$ disease. PET scanning is reported to be more effective than CT scanning in lymph node staging; however, efficacy increases further when results from both methods are combined. ${ }^{3,4}$

Our aim in the present study was to evaluate the need for such routine invasive sampling procedures in all cases to confirm the findings of PET-CT scanning. The question was whether PET-CT scanning was reliable for staging in patients with NSCLC and whether it could decrease the need for mediastinoscopy.

\section{MATERIALS AND METHODS \\ Patient Population}

Seventy-eight consecutive patients with NSCLC who were potential candidates for surgical resection and were admitted to the thoracic surgery unit of our hospital from March 2006 to June 2008 joined this prospective study. 


\section{Abbreviations and Acronyms \\ CT = computed tomography \\ EBUS-FNA = endobronchial ultrasound-guided fine-needle aspiration \\ EUS-FNA = endoscopic ultrasound-guided fine-needle aspiration \\ FDG $=[18 \mathrm{~F}]$-2-fluoro-2-deoxy-D-glucose \\ FNA $\quad=$ fine-needle aspiration \\ MLN = mediastinal lymph node \\ NSCLC $=$ non-small cell lung cancer \\ PET = positron emission tomography \\ SUV $=$ standardized uptake value}

After approval by the institution's ethics committee, oral and written informed consent was obtained from all the subjects. All patients were included in this prospective study, excluding (1) patients with evidence of metastatic disease, except for those with solitary brain or adrenal metastasis; (2) patients who had not undergone PET-CT scanning as part of their preoperative evaluation or who had undergone FDG-PET scanning in another center; and (3) patients with diabetes mellitus whose blood glucose levels could not be controlled and brought to normal values. Because the patients found to have $\mathrm{N} 2$ disease before neoadjuvant therapy were included, we did not include patients receiving neoadjuvant treatment in this study. Complete blood counts and blood chemistry tests, chest radiographs, thoracic CT scans, PET-CT scans, pulmonary function tests, and, if clinically indicated, bone scans and cranial magnetic resonance imaging were performed in all cases. PET-CT scans were performed as part of the prospective studies used to assess the utility of PET-CT scanning in diagnosing or staging tumors. The positive findings on PET-CT and CT scans were labeled to identify the involved lymph node station. Definitive diagnosis was established based on the histopathologic findings of lymph node sampling in mediastinoscopy or biopsy during the surgical procedure. If a mediastinoscopy was performed, histologic evaluation of the specimens was performed with a frozen section. If $\mathrm{N} 2$ disease was present, definitive resection was not performed at that time. These patients received neoadjuvant therapy.

Patients with resectable disease on mediastinoscopy underwent further operative procedures. Three patients underwent transcarinal sleeve pneumonectomy, 16 underwent pneumonectomy (invasion of the left atrium, main pulmonary artery, carina, distal trachea, and proximal main bronchus, with some major fissure invasion), 4 underwent bilobectomy, 2 underwent sleeve lobectomy, and 46 underwent lobectomy. One patient was identified as unresectable (M1) during thoracotomy. Six patients did not undergo thoracotomy because of positive results on mediastinoscopy. One patient given a positive diagnosis after mediastinoscopy underwent resection as a result of drainage to the pleural space caused by tumor necrosis and hemoptysis.

Multistation nodal mediastinal sampling was performed, with removal of levels $2,4,7,8$, and 9 on the right side. For left-sided tumors, lymph nodes at levels 5 and 6 were dissected also. However, nonpalpable station $2 \mathrm{~L}$ could not be removed in some patients. Hilar lymph nodes were also dissected.

Preoperative staging of the mediastinum with PET-CT scanning was compared with conventional preoperative staging with chest CT scanning; the accuracy of each study was assessed against the pathology results obtained by means of mediastinoscopy or MLN dissection at the time of thoracotomy. All patients underwent tissue sampling of MLNs to compare sampling results with imaging results.

\section{Thoracic CT Scanning}

CT examinations were performed by using a helical CT scanner (Brilliance 6; Philips Medical Systems, Cleveland, Ohio). Images (7.5 mm thick) were obtained with sections after intravenous injection of 60 to $100 \mathrm{~mL}$ of contrast material (Ultravist 300; Bayer Schering Pharma, Berlin, Germany). The images were read by a radiologist before obtaining a PET-CT scan. The CT scan reader was blinded to the results of the reference tests. MLNs were considered positive if they were larger than $10 \mathrm{~mm}$ in their short-axis diameters.

\section{PET-CT Scanning}

Whole-body PET-CT scanning was performed with Siemens Biograph 2 PET-CT system (Siemens, Munich, Germany). A whole-body acquisition was performed immediately 1 hour after intravenous administration of FDG (11-16 $\mathrm{mCi})$, and images were obtained from the vertex to the upper thigh region. High-quality images were acquired, and semiquantitative measurements of glucose metabolism were obtained. All patients fasted for at least 4 hours before imaging, their fasting blood glucose levels were within the normal range, and none received insulin to return blood glucose to normal levels. The standardized uptake values (SUVs) of hilar lymph nodes and MLNs were determined from the transverse views by the nuclear medicine physician blinded to results of reference tests. Coronal-sagittal images and their correlation with CT scans were used when the exact location was uncertain. Regions of interest were drawn on the images, and semiquantitative SUV measurements were defined as the regional tissue radioactivity concentration normalized for injected dose and body weight. Results of PETCT scans were considered positive in the mediastinum and hilar area that was separate from the primary mass if the SUV in patients suspected to have lymph node metastases was greater than 2.5 .

\section{Mediastinoscopy}

Mediastinoscopy was performed according to the following criteria: (1) ipsilateral or contralateral MLNs with a diameter of greater than $10 \mathrm{~mm}$ in the short axis on the chest CT scan; (2) MLN uptake on the PET-CT scan; (3) a histopathology of adenocarcinoma on bronchoscopy or transthoracic biopsy; (4) central tumors (tumor involving hilar structures, such as the main bronchus, distal trachea, and main pulmonary artery; visible by means of bronchoscopy; or both), even if $\mathrm{N} 2$ disease was not detected in radiologic examinations; and (5) high-risk surgical candidacy. Mediastinoscopy was not performed in patients excluded from these criteria or found to have N2 disease by means of transbronchial fine-needle aspiration.

In mediastinoscopy biopsy specimens were taken from nodal stations, with $4 \mathrm{~L}, 4 \mathrm{R}$, and 7 being the most commonly sampled. All lymph node stations observed on PET-CT scans were sampled by means of mediastinoscopy. Video-assisted thoracoscopy was performed in patients with positivity of lymph nodes at station 5 , as well when the cervical mediastinoscopy result was negative. In patients with clinical stage I or II NSCLC and without the above criteria based on PET-CT and CT scanning, mediastinoscopy was not performed, but multinodal mediastinal sampling at the time of thoracotomy was performed.

If metastasis of NSCLC to the MLNs was detected at the time of mediastinoscopy, then lung resection was not performed, and patients were referred to neoadjuvant therapy. If the MLNs were negative for metastasis, we continued the procedure with thoracotomy and resection with mediastinal lymphadenectomy.

\section{Statistical Analysis}

CT and PET-CT findings were compared with histopathologic findings in the lymph node stations undergoing biopsy, resection, or both to determine their diagnostic capabilities. The diagnostic efficacy of the CT and PET-CT scans was calculated with sensitivity, specificity, positive and negative predictive values, and accuracy. True positivity and true negativity were assessed based on the presence of N2 disease in patients with NSCLC.

\section{RESULTS}

There were 73 male and 5 female patients (age range, 44 79 years; mean age, 61.3 years) in the study. 
TABLE 1. Sensitivity, specificity, PPV, NPV, and accuracy of MLN metastases on PET-CT and CT scanning of patients with NSCLC

\begin{tabular}{lccccc}
\hline & Sensitivity (TP/TP + FN) & Specificity (TN/TN + FP) & PPV (TP/TP + FP) & NPV $($ TN/TN + FN) & Accuracy $($ TP + TN/total) \\
\hline PET-CT & $81.8 \%$ & $89.5 \%$ & $56.2 \%$ & $96.7 \%$ & $88.4 \%$ \\
CT & $45.4 \%$ & $80.5 \%$ & $27.7 \%$ & $90 \%$ & $75.6 \%$ \\
\hline
\end{tabular}

$P P V$, Positive predictive value; $N P V$, negative predictive value; $M L N$, mediastinal lymph node; $P E T$, positron emission tomography; $C T$, Computed tomography; $N S C L C$, nonsmall cell lung cancer; $T P$, true-positive; $F N$, false-negative; $T N$, true-negative; $F P$, false-positive.

\section{Histopathology}

Final histology was available on 397 lymph node stations (265 N2 tumors from 78 patients, 107 hilar tumors from 71 patients, and $25 \mathrm{~N} 3$ tumors from 33 patients) sampled during mediastinoscopy or surgical intervention. The specific histologic subtypes included adenocarcinoma $(n=16)$, epidermoid carcinoma $(n=41)$, adenosquamous carcinoma $(\mathrm{n}=5)$, and large cell or undifferentiated carcinoma $(\mathrm{n}=$ 16). Pathologic stages of the patients were as follows: 5 stage IA, 20 stage IB, 1 stage IIA, 16 stage IIB, 16 stage IIIA, 16 stage IIIB, and 4 stage IV cases. One of the patients with stage IV disease did not undergo resection because of a nodule identified in the lesion-free lobe during the operation, and 3 other patients with solitary brain metastasis or adrenal metastasis underwent resections.

\section{Thoracic CT Scanning}

All patients underwent chest CT and PET-CT scanning, and the results were compared with the histologic sampling results of mediastinoscopy, mediastinal lymphadenectomy, or both. Comparison of the results of CT scanning and definitive histopathology revealed true-positive $(n=5)$, true-negative $(\mathrm{n}=54)$, false-negative $(\mathrm{n}=6)$, and false-positive $(\mathrm{n}=13)$ results. Enlarged MLNs on CT scans were found in 18 patients, but only 5 of them had N2 disease at pathologic staging. Sixty patients did not have enlarged MLNs; unexpected $\mathrm{N} 2$ disease was found in 6 of them. Of the 11 patients with $\mathrm{N} 2$ disease, $\mathrm{CT}$ scanning correctly identified only $5(45.4 \%)$. Sensitivity, specificity, and positive and negative predictive values of $\mathrm{N} 2$ involvement in thoracic $\mathrm{CT}$ scanning were $45.4 \%, 80.5 \%, 27.7 \%$, and $90 \%$, respectively. The accuracy of CT scanning was $75.6 \%$ (Table 1).

\section{PET-CT Scanning}

PET-CT scanning correctly staged disease in $69(88.4 \%)$ of 78 patients in our study. In patients with FDG uptake into the mediastinum, the presence of N2 disease in 9 of 16 positive PET-CT scans was confirmed by means of mediastinoscopy, surgical intervention, or both. There were 2 false-negative PET-CT scan results among 11 patients with proved $\mathrm{N} 2$ disease. In 62 patients there was no suspicion of MLNs on the PET scans; 2 of these patients had MLNs at pathologic staging. Seven of 16 patients having uptake in MLNs on PET-CT scanning had false-positive results. In 1 of these patients, histopathologic examination revealed lymph node tuberculosis. The characteristics of these patients are presented in Table 2. Thus the sensitivity, specificity, and positive and negative predictive values of MLN involvement in PET-CT scanning were $81.8 \%$, $89.5 \%, 56.2 \%$, and $96.7 \%$, respectively (Table 1 ). McNemar's test performed between CT and PET-CT scans did not yield statistically significant results $(P>.05)$.

Because of the importance of identifying patients with stage IIIA disease properly before lung resection, the records of 2 patients with PET-CT scans that were false-negative for the MLNs were reviewed. These 2 patients underwent surgical resection with mediastinal lymphadenectomy. In pathologic evaluations both patients had metastasis in a paraesophageal lymph node (station 8) and in a single station. These 2 patients had stage T2 epidermoid carcinoma, with 1 nodal station positive for metastatic disease (Table 3). One of the patients had a right hilar and the other had a left lower lobe tumor. The first had mediastinoscopy, with negative results when thoracotomy/pneumonectomy (because of fissural invasion) was performed. The other patient directly had thoracotomy/lobectomy.

There was 52 true-negative, 1 false-negative, 5 true-positive, and 2 false-positive results among 60 patients who had no lymph node enlargement on CT scans, whereas 8 truenegative, 1 false-negative, 4 true-positive, and 5 false-positive results were found in patients with PET-CT scans who had enlarged lymph nodes on CT scans.

Involvement of hilar lymph nodes was assessed in $71 \mathrm{pa}-$ tients after excluding 6 patients identified as having MLN involvement, who were not sent for surgical intervention, as well as 1 patient identified as being unresectable during surgical intervention. On PET-CT scanning, uptake in hilar lymph nodes was detected in 14 patients. Five of these patients had false-positive results. Thirty-nine patients did

TABLE 2. False-positive PET-CT findings of MLNs in patients with NSCLC

\begin{tabular}{lcccc}
\hline Patient no. & Age/sex & Cell type & Station & $\begin{array}{c}\text { Primary } \\
\text { tumor stage }\end{array}$ \\
\hline 1 & $72 / \mathrm{M}$ & Epidermoid Carcinoma & $4 \mathrm{~L}$ & $\mathrm{~T} 2$ \\
2 & $57 / \mathrm{M}$ & Epidermoid Carcinoma & $4 \mathrm{~L}$ & $\mathrm{~T} 2$ \\
3 & $68 / \mathrm{M}$ & Epidermoid Carcinoma & $4 \mathrm{R}$ and 7 & $\mathrm{~T} 2$ \\
4 & $50 / \mathrm{M}$ & Adenocarcinoma & 3 & $\mathrm{~T} 3$ \\
5 & $65 / \mathrm{M}$ & Epidermoid Carcinoma & $4 \mathrm{~L}$ & $\mathrm{~T} 3$ \\
6 & $69 / \mathrm{M}$ & Epidermoid Carcinoma & 5 & $\mathrm{~T} 3$ \\
7 & $66 / \mathrm{M}$ & Epidermoid Carcinoma & $4 \mathrm{~L}$ & $\mathrm{~T} 2$ \\
\hline
\end{tabular}

PET, Positron emission tomography; $C T$, Computed tomography; NSCLC, non-small cell lung cancer; $M$, male. 
TABLE 3. False-negative PET-CT findings of MLNs in patients with NSCLC

\begin{tabular}{llccc}
\hline Patient no. & Age/sex & Cell type & Station & $\begin{array}{c}\text { Primary } \\
\text { tumor stage }\end{array}$ \\
\hline 1 & $71 / \mathrm{M}$ & Epidermoid Carcinoma & 8 & $\mathrm{~T} 2$ \\
2 & $59 / \mathrm{F}$ & Epidermoid Carcinoma & 8 & $\mathrm{~T} 2$ \\
\hline
\end{tabular}

PET, Positron emission tomography; $C T$, Computed tomography; $M L N s$, mediastinal lymph nodes; NSCLC, non-small cell lung cancer; $M$, male; $F$, female.

not have any involvement, and 14 of these patients had falsenegative results. Thus sensitivity, specificity, and positive and negative predictive values of hilar lymph node involvement in PET-CT scans were $34.6 \%, 88.8 \%, 64.2 \%$ and $70.1 \%$, respectively. The accuracy was calculated as $69 \%$ (Table 4).

When hilar lymph node and MLN evaluations based on PET-CT scans were compared with results after resection with mediastinoscopic biopsy, thoracotomy, or both, 16 cases were upstaged (false-negative 2 cases intersected in mediastinal and hilar lymph node assessment), 12 patients were downstaged, and 50 patients remained with the same stage.

\section{Mediastinoscopy}

Mediastinoscopy was performed in 33 of the 78 study patients. The mean lymph node station number per patient after mediastinoscopy was $3.12 \pm 0.78$ and that after thoracotomy was $1.51 \pm 0.53$ and $3.38 \pm 1.45$ for hilar lymph nodes and MLNs, respectively. Mediastinoscopic frozen sections were negative for metastatic disease in 26 patients, who then went on to thoracotomy according to our previous study. ${ }^{5}$ One patient with negative results for frozen section specimens after mediastinoscopy underwent resection, and definitive histopathologic examination of the same sections was positive. This patient was evaluated as having a false-negative result in mediastinoscopic frozen section examination. Seven patients had stage $\mathrm{N} 2$ disease diagnosed by means of mediastinoscopy/thoracoscopy. Six of these were referred to neoadjuvant therapy. One had resection as a result of tumor necrosis and hemorrhage. Two patients were identified as having positive results for station 5 in PET-CT scans and underwent video-assisted thoracoscopy; one of these went to resection with negative results, and the other was referred to neoadjuvant treatment with positive results. In our study, no N3 disease was found in patients who underwent mediastinoscopy. Mediastinoscopy was $96.9 \%$ accurate (1 false-negative result) because the results of the positive frozen sections were confirmed by final pathology, and the results of the negative mediastinoscopy findings were confirmed by mediastinal lymphadenectomy during thoracotomy.

\section{DISCUSSION}

We observed that PET-CT scanning has high reliability in excluding $\mathrm{N} 2$ disease, and mediastinoscopy might not be performed in cases with PET-CT scanning-negative MLNs.
TABLE 4. Sensitivity, specificity, PPV, NPV, and accuracy of hilar lymph node metastases on PET-CT scanning in patients with NSCLC

\begin{tabular}{lccccc}
\hline & Sensitivity & Specificity & PPV & NPV & Accuracy \\
\hline PET-CT & $34.6 \%$ & $88.8 \%$ & $64.2 \%$ & $70.1 \%$ & $69 \%$ \\
\hline$P P V$, Po
\end{tabular}

$P P V$, Positive predictive value; $N P V$, negative predictive value; $P E T$, positron emission tomography; $C T$, computed tomography; $N S C L C$, non-small cell lung cancer.

Hellwig and coworkers ${ }^{6}$ stated that the choice of an SUV of 2.5 as the threshold is justified for mediastinal staging because false-negative results plus false-positive results is minimized. The resulting high negative predictive value of 96\% allows the omission of mediastinoscopy in patients with negative mediastinal findings on FDG-PET images. However, Bryant and colleagues ${ }^{7}$ have used an SUV of 5.3 instead of the traditional value of 2.5 , and the accuracy of integrated PET-CT scanning for each N2 nodal station has been maximized and at least $92 \%$ for each. We used the 2.5 cutoff rate as a positive PET-CT result for the mediastinal and hilar lymph nodes. We do not have enough patients for evaluating different SUV cutoff rates.

In predicting the mediastinal contents, PET scanning is reported to be superior to $\mathrm{CT}$ scanning. In a meta-analysis including 40 studies, the sensitivity and specificity were reported as $85 \%$ and $90 \%$ for PET-CT scanning versus $61 \%$ and $79 \%$ for CT scanning, respectively. ${ }^{8}$ In a study by Gupta and associates, ${ }^{4}$ the sensitivity, specificity, and accuracy of PET scanning in identifying MLNs was reported as $87 \%, 91 \%$, and $82 \%$, respectively, and corresponding values for CT scanning were $68 \%, 61 \%$, and $63 \%$.

It is possible to decrease false-negative and false-positive results by combining PET and CT scanning in identifying metastatic lymph nodes. Weng and coworkers ${ }^{9}$ reported that to contribute to each other, imaging methods should be used together and that this should reveal better results for sensitivity, specificity, and accuracy $(82 \%, 96 \%$, and $91 \%$, respectively) and that be more accurate in the staging of mediastinal disease. Cerfolio and colleagues ${ }^{10}$ reported that in the evaluation of N2 disease, PET-CT scanning was more accurate than PET scanning alone (96\% vs $93 \%$ ). A study by Kelly and associates ${ }^{3}$ concluded that adding PET scanning to CT scanning used for clinical staging of patients with NSCLC would improve the identification of $\mathrm{N} 2$ disease in the preoperative period. When the results of PET and CT scanning were combined in this study, sensitivity, specificity, and accuracy rates were improved $(70 \%$, $98 \%$, and $91 \%$, respectively). In our study the results for MLN staging by means of PET-CT scanning were superior to those for CT scanning. The sensitivity, specificity, and positive and negative predictive values of MLN involvement in PET-CT scanning were $81.8 \%, 89.5 \%, 56.2 \%$, and $96.7 \%$, and these values in thoracic CT scanning were $45.4 \%, 80.5 \%, 27.7 \%$, and $90 \%$, respectively. Mediastinoscopic biopsy or surgical resection was taken as the basis for 
the evaluation of the cases. Not performing mediastinoscopy in patients with negative lymph nodes as shown by $\mathrm{CT}$ and PET-CT scanning can be comprehended as a verification bias. However, in $91 \%$ of the cases, lymph node evaluation was performed histopathologically on those nodes resected surgically without taking mediastinoscopy into consideration. For this reason, we think that bias is not an issue in the analysis of our data.

Gould and colleagues ${ }^{8}$ reported that PET scanning was more sensitive but less specific when CT scanning showed enlarged lymph nodes than when CT scanning showed no lymph node enlargement. We found 1 false-negative and 8 true-negative results in patients who had lymph node enlargement on CT scanning with the evaluation of PET-CT scanning. The sensitivity and specificity of PET-CT scanning in our patients with enlarged lymph nodes on CT scanning was $80 \%$ and $61.5 \%$, respectively, whereas the values were $83.3 \%$ and $96.2 \%$ in patients who had no lymph node enlargement. Because of the poor adjunct of PET-CT scanning, in patients with enlarged lymph nodes on CT scanning, mediastinoscopy can be considered as a first alternative for mediastinal staging. With the consideration of intelligible spending of the resources allocated to health, in patients who had N0 disease mediastinoscopically, the use of PET scanning can be limited to demonstrating distant metastases.

Kelly and associates ${ }^{3}$ found lower PET scanning sensitivity rates than those reported in the literature, especially for patients with adenocarcinoma $(62 \%)$. Four of 5 patients having false-negative PET scan results for mediastinal evaluation had adenocarcinoma as the histologic type. Patients with false-negative lymph node results on PET scanning can end in incomplete resection if the surgeon abides by clinical staging. In our study the number of patients with falsenegative results on mediastinal evaluation with PET-CT scanning was very low and limited to 2 . Both of these patients had epidermoid carcinoma, involvement was at station 8 , and there was a single involvement. Because it is not possible to reach this station with mediastinoscopy, in one of these patients N2 disease could not be diagnosed with mediastinoscopy. Endoscopic ultrasound-guided fine-needle aspiration (EUS-FNA) is a minimally invasive technique used for staging of lung cancer and allows reaching the lymph node stations numbered 7 and 8 and especially 9 , which cannot be approached easily by using standard mediastinoscopy. ${ }^{11}$ According to Eloubeidi and coworkers, ${ }^{12}$ EUS-FNA is more accurate $(92.5 \%)$ and has a higher positive predictive value $(100 \%)$ than PET scanning for posterior MLNs. DeLeyn and associates, ${ }^{13}$ on the other hand, stated that endobronchial ultrasound-guided fine-needle aspiration (EBUS-FNA) and EUS-FNA have high specificities but low negative predictive values; therefore in patients with negative results obtained with these techniques, invasive surgical techniques are indicated. We did not use EUS-FNA and EBUS-FNA because they were not available in our hospital. In patients undergoing neoadjuvant treatment, remediastinoscopy is recommended to evaluate the response to treatment, despite it being technically more challenging than the initial procedure. In our clinical practice we make use of remediastinoscopy for mediastinal staging after neoadjuvant treatment. However, we did not include patients receiving neoadjuvant treatment in this study. For restaging the mediastinum, as an alternative to this technique, EBUS-FNA and EUS-FNA are recommended as invasive tests yielding similar results to video-assisted thoracoscopic surgery and remediastinoscopy. ${ }^{13}$ In a series of 124 patients receiving neoadjuvant treatment for $\mathrm{N} 2$ disease and undergoing mediastinal restaging with EBUS-FNA, the specificity was high, but the negative predictive value was low $(20 \%)$, and negative tumor findings had to be confirmed with surgical staging. ${ }^{14}$

If clinical staging is taken as the basis, patients with falsepositive MLN results can receive unnecessary chemotherapy before curative surgical intervention. In our study 13 patients with enlarged lymph nodes on CT scanning were evaluated as having false-positive results, and 5 of these patients had lymph node abnormality with false-positive enhancement on PET-CT scanning. Two other patients identified as having false-positive results on PET-CT scanning were found to have lesions smaller than $10 \mathrm{~mm}$ on CT scanning. All of these patients were evaluated with mediastinoscopy, and they were identified as having negative results for N2 disease (1 patient was further examined with videoassisted thoracoscopy) and underwent resection. Of 7 patients identified as having false-positive results on PETCT scanning, 6 had epidermoid carcinoma as the cellular type. In 4 of these patients, the primary tumor was pT2, and in the remaining 3 patients, it was pT3. There are publications claiming that false-positive appearance of lymph nodes in PET scanning can be due to inflammatory or granulomatous processes. ${ }^{15,16}$ In our study of patients with falsepositive results on PET-CT scanning, only 1 was identified to have tuberculosis, and in the remaining patients, histopathologic examination of the lymph nodes was reported as a reactive change.

With PET scanning, it can be difficult to localize the lymph nodes in the hilar region and to differentiate them from MLNs. Scott and associates ${ }^{17}$ made a retrospective analysis of 25 patients with potentially operable NSCLC evaluated for N2 disease with PET scanning and found that extensive hilar metastases were wrongly classified as mediastinal because of their proximity to the mediastinum. To surmount this problem, Cerfolio and colleagues ${ }^{10}$ used PET-CT scanning to evaluate hilar lymph nodes and had significantly better results compared with those obtained with PET scanning alone. In their PET-CT evaluation, sensitivity, specificity, and positive and negative predictive values were $94 \%, 89 \%, 43 \%$, and $99 \%$ respectively. With the PET-CT scanning used in our study, it was 
possible to localize hilar lymph nodes; however, in particular false negativity was high (17/71) and other parameters were low, including sensitivity $(34.6 \%)$.

A relatively small number of patients is the limitation of this study. However, we concluded that PET-CT scanning yields better results than $\mathrm{CT}$ scanning. Negative appearances of MLNs in PET-CT scanning results in high success in predicting the mediastinal content; in positive appearances the success of prediction is limited. Therefore there is the need for mediastinoscopy in PET-CT scanning-positive MLNs, but it might not be necessary for PET-CT scanning-negative lymph nodes.

\section{References}

1. Graeter TP, Hellwig D, Hoffmann K, Ukena D, Kirsch CM, Schäfers HJ. Mediastinal lymph node staging in suspected lung cancer: comparison of positron emission tomography with F-18-fluorodeoxyglucose and mediastinoscopy. Ann Thorac Surg. 2003;75:231-5.

2. Roth JA, Atkinson EN, Fossella F, Komaki R, Bernadette Ryan M, Putnam JB Jr, et al. Long-term follow-up of patients enrolled in a randomized trial comparing perioperative chemotherapy and surgery with surgery alone in resectable stage IIIA non-small-cell lung cancer. Lung Cancer. 1998;21:1-6.

3. Kelly RF, Tran T, Holmstrom A, Murar J, Segurola RJ Jr. Accuracy and cost-effectiveness of [18F]-2-fluoro-deoxy-D-glucose-positron emission tomography scan in potentially resectable non-small cell lung cancer. Chest. 2004;125:1413-23.

4. Gupta NC, Tamim WJ, Graeber GG, Bishop HA, Hobbs GR. Mediastinal lymph node sampling following positron emission tomography with fluorodeoxyglucose imaging in lung cancer staging. Chest. 2001;120:521-7.

5. Sanli M, Isik AF, Tuncozgur B, Akar E, Deniz H, Bakir K, et al. The reliability of mediastinoscopic frozen sections in deciding on oncological surgery in bronchogenic carcinoma. Adv Ther. 2008;25:488-95.

6. Hellwig D, Graeter TP, Ukena D, Groeschel A, Sybrecht GW, Schaefers HJ, et al. 18F-FDG PET for mediastinal staging of lung cancer: which SUV threshold makes sense? J Nucl Med. 2007;48:1761-6.
7. Bryant AS, Cerfolio RJ, Klemm KM, Ojha B. Maximum standard uptake value of mediastinal lymph nodes on integrated FDG-PET-CT predicts pathology in patients with non-small cell lung cancer. Ann Thorac Surg. 2006;82: 417-22.

8. Gould MK, Kuschner WG, Rydzak CE, Maclean CC, Demas AN, Shigemitsu H, et al. Test performance of positron emission tomography and computed tomography for mediastinal staging in patients with non-small-cell lung cancer: a meta analysis. Ann Intern Med. 2003;139:879-92.

9. Weng E, Tran L, Rege S, Safa A, Sadeghi A, Juillard G, et al. Accuracy and clinical impact of mediastinal lymph node staging with FDG-PET imaging in potentially resectable lung cancer. Am J Clin Oncol. 2000;23:47-52.

10. Cerfolio RJ, Ojha B, Bryant AS, Raghuveer V, Mountz JM, Bartolucci AA. The accuracy of integrated PET-CT compared with dedicated PET alone for the staging of patients with nonsmall cell lung cancer. Ann Thorac Surg. 2004;78: 1017-23.

11. Eloubeidi MA. Endoscopic ultrasound-guided fine-needle aspiration in the staging and diagnosis of patients with lung cancer. Semin Thorac Cardiovasc Surg. 2007;19:206-11.

12. Eloubeidi MA, Cerfolio RJ, Chen VK, Desmond R, Syed S, Ojha B. Endoscopic ultrasound-guided fine needle aspiration of mediastinal lymph node in patients with suspected lung cancer after positron emission tomography and computed tomography scans. Ann Thorac Surg. 2005;79:263-8.

13. De Leyn P, Lardinois D, Van Schil PE, Rami-Porta R, Passlick B, Zielinski M et al. ESTS guidelines for preoperative lymph node staging for non-small cell lung cancer. Eur J Cardiothorac Surg. 2007;32:1-8.

14. Herth FJ, Annema JT, Eberhardt R, Yasufuku K, Ernst A, Krasnik M, et al. Endobronchial ultrasound with transbronchial needle aspiration for restaging the mediastinum in lung cancer. J Clin Oncol. 2008;26:3346-50.

15. Steinert HC, Hauser M, Allemann F, Engel $H$, Berthold $T$, von Schulthess GK, et al. Non-small cell lung cancer: nodal staging with FDG PET versus CT with correlative lymph node mapping and sampling. Radiology. 1997;202:441-6.

16. Roberts PF, Follette DM, von Haag D, Park JA, Valk PE, Pounds TR, et al. Factors associated with false-positive staging of lung cancer by positron emission tomography. Ann Thorac Surg. 2000;70:1154-9.

17. Scott WJ, Schwabe JL, Gupta NC, Dewan NA, Reeb SD, Sugimoto JT. Positron emission tomography of lung tumors and mediastinal lymph nodes using [18F]fluorodeoxyglucose. The Members of the PET-Lung Tumor Study Group Ann Thorac Surg. 1994;58:698-703. 